\title{
Literate und narrative Textgestaltung in der Zweitsprache zwischen Mündlichkeit und Schriftlichkeit - Grundschüler türkischer Herkunftssprache erzählen einen Stummfilm auf Deutsch
}

\begin{abstract}
Während die Entwicklung narrativer Kompetenzen in gesprochener Sprache häufig als Voraussetzung literaler Kompetenzen gesehen wird, stellt der Beitrag die strukturelle Verschiedenheit beider Kompetenzbereiche heraus. Dabei wird an der mehrfach kritisierten Vorstellung eines Kontinuums zwischen einem oraten und einem literaten Pol sprachstruktureller Mittel ebenso festgehalten wie an der analytischen Trennung zwischen einer medialen und einer sprachstrukturellen Dimension. Am Beispiel mündlicher und schriftlicher Erzählungen eines Stummfilms durch Erstklässler türkischer Herkunft in ihrer Zweitsprache Deutsch werden zunächst interaktive Prozesse der Textproduktion unter den Bedingungen des Diktierens und des kooperierenden Schreibens rekonstruiert, aus denen sich zwei unterschiedliche Formen eines Scaffolding durch erwachsene Interaktionspartner ergeben. Es schlieBen sich eine orat-literat-Analyse des Gebrauchs von Nominalphrasen in den Texten sowie narrative Analysen an, die die Realisierung globaler Erzählstrukturen, die sprachliche Gestaltung der Wiederaufnahme und den Gebrauch direkter und indirekter Figurenrede untersuchen. Die Verteilung der Texte entlang der beiden Kontinua zeigt ein partielles Auseinandertreten der narrativen und literaten Indikatoren, die jeweils stärker in den mündlichen bzw. schriftlichen Texten in Erscheinung treten. Im Fazit wird der Beitrag medialer Strukturierungshilfen für die konzeptuelle Schreibaufgabe herausgearbeitet.
\end{abstract}

\section{Mediale, narrative und literale Kompetenzen im ersten Schuljahr}

In der neueren Diskussion zum Erwerb literater Kompetenzen im Vor- und Grundschulalter wird von einer engen Beziehung zwischen literaten und narrativen Fähigkeiten ausgegangen (Andresen 2005, 2011; Dehn 2011; Feilke 2007; Maas 2008, 2010; Merklinger 2011; Ulich 2003). Die Entstehung satzübergreifender Diskurseinheiten in gesprochener Sprache erscheint als wichtige Ressource zum Schreiben von Texten, die über einfache Listen oder die Gestaltung einzelner Sprechakte im Medium der Schrift hinausgehen. Mögliche Ausgangspunkte hierfür sind die Rezeption vorgelesener Schriftsprache in Bilderbuchinteraktionen (Lonigan/Whitehurst 1998; Hargrave/Sénéchal 2000; Kraus 2005), die Teilhabe an mündlichen 
Erzählsituationen mit Erwachsenen und älteren Kindern oder Rollenspiele (Andresen 2002), in denen fiktionale Kontexte sprachlich hergestellt werden.

Maas (2008, S. 335) gebraucht die Metapher des Bootens oder Bootstrappings für den Zusammenhang beider Kompetenzbereiche, denen dadurch aber auch unterschiedliche Ebenen der Komplexität zugewiesen werden: Mithilfe des einfacheren Programms der Erzählinteraktion werde das komplexere der Literalität gestartet. Dabei geht er aber von einem skalaren Konzept von Literalität aus, an dessen Polen sich orate und literate Strukturen gegenüberstehen. Zu den wichtigsten Parametern der Literalität, die Maas auf der sprachstrukturellen Ebene verankert, zählen syntaktische Integration und lexikalische Explizitheit.

In ihrem mit einem anthropologischen Gegensatz von Nähe- und Distanzsprache argumentierenden Modell stellen Koch/Oesterreicher (1985, 2007) zehn grundlegende Parameter auf, die zwischen ihren extremen Ausprägungen ebenfalls Kontinua, vor allem aber Ungleichzeitigkeiten aufweisen. Der Entwicklungsprozess des ,Distanzsprechens' setzt zwar zunächst enge Vertrautheit der Interaktionspartner am ,Nähepol' des Kontinuums voraus. Die in diesem Rahmen entstehende Interaktion entwickelt sich dann in Richtung monologisches Sprechen und Referenz außerhalb des gemeinsamen raum-zeitlichen Kontexts (,Distanzpol'), lange bevor andere Parameter des Distanzpols wie die Antizipation des, generalisierten Lesers' oder die Themenentfaltung entlang einer bestimmten Textsorte erreicht werden.

Feilke (2007, S. 34) hat eine deutliche Kritik dieses Kontinuummodells von Literalität formuliert. Danach müsse ein Schrifttext ,[...] in jedem Fall den Bedingungen konzeptioneller Schriftlichkeit genügen, wenn eine Form der Distanzkommunikation vorliegt“. Das Beispiel des Gebrauchs von direkter oder indirekter Rede in fiktionalen Texten, in dessen Kontext er diese These vorträgt, spricht dagegen bereits selbst gegen diese dichotomische Sichtweise: Es gibt in fiktionalen Texten eben sehr unterschiedliche Möglichkeiten, die Redewiedergabe von Protagonisten zu gestalten, die vielleicht mehr oder weniger literat sind, aber gewiss nicht einfach den Anforderungen der Textsorte genügen oder nicht genügen.

Die unterschiedlichen literalen Textwelten und Diskurstraditionen, denen - bei Beherrschung ihrer formalen Mittel - konzeptionelle Schriftlichkeit zugesprochen wird, liegen selbst auf unterschiedlichen Stufen innerhalb dieses Kontinuums, je nachdem, welche sprachstrukturellen Indikatoren hierfür veranschlagt werden. Gemessen am Merkmal der syntaktischen Integration liegen Gesetzestexte weit näher am literalen Pol als etwa Märchen, wie dies innerhalb des fiktionalen Genres für den Gebrauch der indirekten Rede gegenüber der direkten gilt. In der Faktoranalyse eines großen Korpus gesprochener und geschriebener Sprache am Beispiel des Englischen hat Biber (1988) verschiedene Parameter ermittelt, auf denen dieselben Texte jeweils 
unterschiedlich positioniert sein können. Der Parameter des Narrativen liegt dort quer zu den Skalen, in denen sich orate und literate Strukturen gegenüberstehen. Mithilfe sprachstruktureller Indikatoren lässt sich also nicht nur die Idee eines Kontinuums von sprachlichen Äußerungen/Texten begründen, sondern auch das Auseinandertreten genuin literaler und narrativer Indikatoren, wie dies im Folgenden am Beispiel von Schülertexten aus dem ersten Schuljahr untersucht werden soll.

An der Idee des Kontinuums von oraten und literaten Strukturen muss aber auch aus sprachdidaktischer Perspektive festgehalten werden: Sprachlerner erwerben die entsprechenden sprachlichen Strukturen über zahlreiche Zwischenstufen, in denen die Ansprüche Erwachsener an die entsprechende Gestaltung der Texte noch unvollständig realisiert sind. Dabei können nicht nur Zielkonflikte zwischen einer stärker literaten oder narrativen Ausgestaltung der Texte auftreten; vielmehr werden diese auch noch von den jeweils unterschiedlichen Zwängen des phonischen oder grafischen Mediums überlagert.

Eine ganze Reihe narrativer Kompetenzen kann weder einfach in das andere Medium übertragen werden, noch den Anforderungen der Kontextentbindung und des Bezugs auf einen generalisierten Leser gerecht werden. Es ist das Verdienst der neueren linguistischen Erzählforschung, die gesprochene Sprache in Alltagskontexten zum Ausgangspunkt nahm (Labov/ Waletzky 1967; Labov 1972; Quasthoff 1980; Heath 1983), gerade die besonderen Leistungen dieser Erzähler demonstriert zu haben, die durch die Brille eines literarischen Erzählens weder in ihrer komplexen Struktur noch in ihrer Funktionalität erkannt wurden. Nicht zuletzt in der neueren Literatur selbst spielt die Figur des begnadeten, aber illiteraten Erzählers eine wichtige Rolle. In ihrer Typologie unterschiedlicher Verlaufsformen des Erzählerwerbs in der Grundschule arbeitet etwa Ohlhus (2005) die Unterschiede zwischen einem szenischen und einem elaborierenden Erzähltyp heraus, die beide auch auf unterschiedliche Weise den Wechsel des Mediums verarbeiten.

So nehmen Diskurspartikeln gerade keinen expliziten Bezug auf das von Sprecher und Hörer geteilte Wissen, signalisieren aber vielleicht gerade dadurch in besonders wirkungsvoller Weise unterschiedliche narrative Funktionen (Maas 2010, S. 109 ff.). Wörtliche Rede leistet eine Perspektivierung der Erzählung auf genauso effektive Weise, wenn die unterschiedlichen Sprecherrollen prosodisch markiert werden, wie durch explizite Einbettungen in Einleitungssätze. Die Identifizierung eines Protagonisten bei der Wiederaufnahme kann narrativ genauso wirkungsvoll durch eine Rede signalisiert werden, in der der Bezug zum früheren Geschehen deutlich wird, wie durch eine entsprechende Relativsatzkonstruktion. Umgekehrt führt die Herstellung von Referenzeindeutigkeit, strikter Chronologie und expliziter konnektiver Verknüpfung der Äußerungen vielleicht zu einem literaten, aber 
narrativ nicht sehr wirkungsvollen Text, wie sich nicht zuletzt an den schulisch normativ orientierten Umgestaltungen von Erzähltexten in der Grundschule zeigt.

Aufgrund dieser Wirkungen medialer Faktoren ist die scharfe Trennung von Medium und Konzeption vor allem in Kochs und Oesterreichers Konzept in die Kritik geraten. Die Autoren gehen von der Dichotomie einer grafischen oder phonischen Realisierungsform von Sprache aus, die unabhängig von einer spezifischen Versprachlichungsstrategie auf der Ebene der Konzeption zu analysieren ist. Mit dem Begriff der Skribalität akzentuiert Maas (2008) entsprechend eine reproduzierende Schreibpraxis, die von den höheren literaten Funktionen des Schreibens abgekoppelt sein kann, historisch etwa durch den Gegensatz von Sekretärs- und Autorfunktion in der abendländischen Schriftgeschichte (Ludwig 2005, S. 146170). Ein Schreiber, der den durch den diktierenden Autor phonisch realisierten konzeptuell schriftlichen Text auch grafisch realisiert, braucht nach diesem Verständnis über keine Textkompetenz im konzeptionellen Sinne zu verfügen.

Wrobel (2010, S. 32) spricht in seiner Kritik an Koch und Oesterreicher von der ,Liquidierung des Medialen' und versammelt unterschiedliche Argumente gegen das Nähe-Distanz-Modell, deren Gemeinsamkeit die Rückwirkung medialer Aspekte auf die Konzeption einer Äußerung ist. Ein Teil dieser Kritik bezieht sich allerdings nur auf die Unschärfe der anthropologischen Nähe-Distanz-Parameter, wie sie sich teilweise auch bei Maas (2008) findet. Darüber hinaus greift Wrobel (2010, S. 38) aber auch auf Luhmanns Medientheorie zurück, die von einer strikten Differenz und Unübersetzbarkeit beider Formen ausgehe. Die Schwäche dieser Argumentation liegt wiederum in der Unterstellung einer bloßen Äußerlichkeit des Mediums. Die Unterscheidung ist dagegen nur als analytische sinnvoll: Keine sprachliche Äußerung kann unabhängig von einem bestimmten Medium bestehen, aber in der Analyse ihrer sprachlichen Form lassen sich durchaus mediale und strukturelle Aspekte voneinander trennen. Dieses zeigt sich - und soll im Folgenden auch herausgearbeitet werden - nicht zuletzt bei Schreibanfängern, deren medial schriftliche Realisierung eines Textes Planungskapazitäten von der strukturell-konzeptuellen Ebene abzieht.

Im ersten Schuljahr überlagern sich beide Kompetenzbereiche in besonderer Weise mit der medialen Dimension. Auch wenn vereinfachenden Modellierungen, die den Erwerb basaler Schreibfähigkeit von der Textkompetenz abkoppeln (Whitehurst/Lonigan 1998), nicht zuzustimmen ist und also der Text - auf allen seinen Ebenen - von Anfang an als Herausforderung für den Schreiber präsent ist (Weinhold 2000), spielt die Bewältigung des Mediums Schrift allein schon quantitativ eine viel entscheidendere Rolle. Um Schreibanfänger zunächst von den medialen Aufgaben der Schrift zu entlasten, hat Merklinger (2011) das experimentelle Verfahren des Diktie- 
rens in einer Weise weiterentwickelt, die den Kindern in einer dialogischen Situation bereits einige mediale, aber vor allem konzeptuelle Besonderheiten des Schreibens erfahrbar macht. Der Einfluss dieses Designs auf Elemente der Textstruktur soll dabei ebenso berücksichtigt werden wie seine Wirkung auf die narrative Gestaltung.

Im Folgenden soll es darum gehen, an fünf Fallstudien herauszuarbeiten, wie Erstklässler mit Türkisch als Erstsprache im Übergang von Mündlichkeit und Schriftlichkeit in der Zweitsprache Deutsch auf unterschiedliche Weise den partiellen Gegensatz narrativer und literater Strukturen bearbeiten. Als Textgenre wurde mit einem Stummfilm ein reproduktives gewählt, das zwar im Sinne einer schulisch erwarteten ,literaten 'Textsorte höhere Anforderungen stellt als die Erlebniserzählung, andererseits weniger auf die Ko-Konstruktion von kindlichem Erzähler und erwachsenem Zuhörer angewiesen ist (Hausendorf/Quasthoff 2005; Kern/Quasthoff 2005; Becker 2001).

Nach einer Vorstellung des Projektkontextes, aus dem die Daten entstammen (Teil 2), werden zunächst die interaktiven Entstehungskontexte von zwei der später analysierten Texte analysiert, bei denen ein dem MerklingerDesign verwandtes Verfahren des Diktierens gewählt, aber durch den anschließenden Wechsel zur Schreiber- bzw. Autorenrolle weiterentwickelt wurde (Teil 3). So eröffnen sich Einblicke in Praktiken von Erstklässlern, die unter den medialen Anforderungen des Schreibens auch Probleme der Textstruktur bearbeiten. Es folgt eine Auswertung der gesprochenen und geschriebenen Texte nach dem Verfahren der orat-literat-Analyse (Maas 2010). Schließlich werden einige narrative Aspekte der Texte mithilfe von Indikatoren untersucht, die sich nicht mit den Indikatoren der orat-literatAnalyse decken. Die unterschiedlichen Dynamiken beider Kompetenzbereiche werden am Beispiel der untersuchten Texte in der abschließenden Zusammenfassung herausgearbeitet.

\section{Das Projekt LAS und die Datengrundlage der Analyse}

Die Daten wurden in dem Projekt, Schriftspracherwerb in der Organisation Schule unter den Bedingungen von Migration und Mehrsprachigkeit ${ }^{6}$ (LAS) ${ }^{1}$ erhoben. Dieses interdisziplinäre und international vergleichende Projekt untersuchte jeweils an einer ersten und siebten Klasse im Ruhrgebiet einerseits und in Istanbul andererseits, wie Migrationen und Mehrsprachigkeit und ihre spezifischen Sprachen- und Minderheitenkonstellationen über die

Ich danke Anja Boneß, Helena Olfert und Inken Sürig, die an der Erhebung, Aufbereitung und Auswertung des Materials beteiligt waren, sowie Christoph Schroeder und Yazgül Şimşek für wertvolle Hinweise bei der Analyse der Texte. 
sprachlich-kulturelle Ausstattung einzelner Schülerinnen und Schüler in die schulische Praxis eingehen. Dabei stehen Schriftspracherwerbsprozesse auf der Ebene basaler Lese- und Schreibkompetenzen einerseits und der Textkompetenz andererseits in einem besonderen Fokus. Berücksichtigt wurde außerdem, wie die schulisch organisierte Vermittlung von Literalität in historisch-kulturelle Wissens- und Sprachauffassungen eingebettet ist. Schulisches Lernen sollte gleichzeitig als ein Beschränkungs- und Ermöglichungszusammenhang in den Blick genommen werden, in dem durch bestimmte soziale Aktivitäten Lernprozesse angestoßen, aber auch blockiert werden, Literalität aber immer durch spezifische Interaktionen hindurch erworben wird, auch wenn sich Schreibkompetenzen in der Perspektive des Heraustretens aus der Interaktion entwickeln (vgl. Mehlem 2010).

Dazu wurden die Schulklassen über einen Zeitraum von neun Monaten wöchentlich besucht, wobei jeweils eine Unterrichtsstunde videografiert und zusätzliche Notizen angefertigt wurden. Das in der Stunde verwendete oder von den Schülern erstellte schriftliche Material wurde soweit möglich kopiert und mit der Videoanalyse in Beziehung gesetzt. Im Fokus der Betrachtung standen jeweils sechs Schüler, deren Erstsprache mit der Unterrichtssprache identisch war (Deutsch als L1 in Deutschland, Türkisch als L1 in der Türkei), und sechs Schüler mit einer von der Schulsprache abweichenden Familiensprache (in Deutschland überwiegend Schüler mit Türkisch als L1; Schüler mit Kurdisch als L1 in der Türkei).

Die Analyse der Unterrichtsinteraktion und der Schreibprodukte der Schüler wurde ergänzt durch sprachliche Tests, die jeweils zu Beginn und zum Ende des Untersuchungszeitraums durchgeführt wurden. Dabei wurden sowohl gesprochene als auch geschriebene Äußerungen, bei den mehrsprachigen Schülern in beiden Sprachen, in verschiedenen Textsorten erhoben. Zu den Analysen des Projektes gehören Fallstudien ein- und mehrsprachiger Schüler, auf die sich die hier vorgestellte Analyse stützt (Boneß et al. i.Vorb.).

Als Impuls für die Nacherzählungen der Kinder und ihre späteren Verschriftungen diente ein Stummfilm, der zu diesem Zweck erstellt wurde. Der fehlende Ton sollte nicht nur die Phantasie der Kinder anregen, sondern auch die wörtliche Wiederholung von Gesagtem ausschließen. Die Geschichte weist die klare Struktur einer Komplikation (eine Frau verliert einen Umschlag mit Geld), eines Konflikts (die beiden Finder streiten darüber, ob sie den Umschlag zurückgeben, also nach ihrem Besitzer suchen sollen) und einer Auflösung auf (die Besitzerin wird gefunden und bedankt sich bei den Findern), erleichtert also die Etablierung einer hierarchischen Erzählstruktur, auch wenn diese in der Regel von Erstklässlern noch nicht erreicht wird (vgl. Boueke et al. 1995).

Der Test wurde zweimal durchgeführt, um Fortschritte in der narrativen und literalen Entwicklung über den Zeitraum von acht Monaten feststellen zu können. Im ersten Testdurchlauf wurden die Kinder nach der 
mündlichen Aufnahme des spontan erzählten Films gebeten, dem Interviewer eine Version des Textes zu diktieren, damit ,man sie später noch einmal lesen kann' nach einer von Pontecorvo und Zucchermaglio (1989) erstmals verwendeten Methode, die in die schriftvorbereitende Praxis vieler Kindergärten Eingang gefunden hat (Ulich 2003) und in den letzten Jahren von Merklinger (2011) und Hüttis-Graff (2011) weiterentwickelt wurde. Im zweiten Testdurchlauf wurde bereits die erste Version diktiert, während nun die zweite direkt vom Schüler aufgeschrieben wurde.

\section{Von der Diktiersituation zur interaktiven Schreibsituation}

Die erste Dimension der Analyse der Schülertexte fokussiert auf den interaktiven Kontext, in dem zunächst der diktierte, dann der geschriebene Text entsteht. Bereits bei der Beschreibung des Untersuchungsdesigns wurde darauf hingewiesen, dass in den beiden Tests ein Diktierdesign verwendet wurde, das mit dem in der Zwischenzeit veröffentlichten von Merklinger (2011) große Ähnlichkeiten aufweist. Unter Bezug auf die dort entwickelten Analysekategorien werden zunächst zwei der Transkripte genauer untersucht (Abschnitt 3.1).

In derselben 1:1-Situation fand nach dem Diktieren ein Rollenwechsel statt: Das diktierende Kind sollte nun selbst die Schreiberrolle übernehmen. Alle Äußerungen von Interviewer und Kind, die diesen Prozess begleiten, wurden ebenfalls aufgezeichnet. Im Design des Projekts war ein vollständig vom Interviewer unabhängiges Schreiben vorgesehen (analog zu den Schreibaufgaben der Schule). Dieses Szenario wurde näherungsweise nicht in allen Texten durchgehalten. Bei zwei Mädchen kam es während des Schreibens zu zahlreichen Rückfragen und Kommentaren bzw. Impulsen, Antworten und Vorschlägen des Interviewers bzw. der Interviewerin. Was als ,Unfall' erschien, kann als eine Verlängerung des Merklinger-Designs in das frühe Schreiben hinein reanalysiert werden: Durch einen Vergleich der Diktier- und der Schreibinteraktionen können unterschiedliche Grade des Heraustretens des Schreibers aus der Interaktion miteinander verglichen werden. Interessant ist dabei vor allem, was die Kinder - im Unterschied zur Diktiersituation - beim eigenen Schreiben thematisieren (Abschnitt 3.2).

\subsection{Interaktionen beim Diktieren}

Das Ziel, die Kinder bereits im Mündlichen Sprachgebrauch zu konzeptueller Schriftlichkeit herauszufordern (Merklinger 2011, S. 82), kann nur erreicht werden, wenn die Skriptorin nicht in einen mündlichen Dialog mit dem Kind eintritt, sondern indem sie die neue Aufgabe medial und konzeptuell zur Geltung bringt. Medial geschieht dies zunächst dadurch, dass sie 
beim Schreiben mitspricht und dadurch dem Kind die Entschleunigung des Schreibprozesses immer wieder signalisiert. Merklinger spricht davon, „die Langsamkeit des Mediums hörbar" zu machen (2011, S. 84). Dieses Design wird durch die Sitzanordnung von Kind und Skriptorin und die Verwendung von Großbuchstaben, die dem Kind den Nachvollzug des Schreibens erleichtert, unterstützt.

Auch in unserem Falle saßen Kind und Skriptor nebeneinander und das Kind konnte sehen, was geschrieben wurde, allerdings wurden nicht nur keine Großbuchstaben verwendet, sondern die Schreiber schrieben auch in ihrer gewöhnlichen Handschrift, was Lesbarkeit für die Kinder weitgehend ausschloss.

Im Umgang mit der Diktiersituation zeigen sich bereits bei den zwei untersuchten Transkripten deutliche Unterschiede: Während $\mathrm{FEH}^{2}$ sich dem Rhythmus des Mitschreibens der Skriptorin anpasst und nur wenig unterbrochen werden muss, um das Aufschreiben ihres Textes zu sichern, reiht SEV mehrmals in schneller Abfolge mehrere Äußerungen hintereinander, sodass der Skriptor beim Mitschreiben an einer deutlich früheren Äußerung ansetzt und einige Äußerungen SEV's gar nicht mitgesprochen werden. Von 23 Äußerungen FEH's spricht die Skriptorin beim Schreiben 17 (73,9\%) mit, während von 28 Äußerungen SEV's nur 14 (50\%) vom Skriptor wiederholt werden, und diese zum Teil mit einer erheblichen Verzögerung. FEH hat sich also bereits viel stärker als SEV den medialen Anforderungen des Schreibprozesses angepasst, was sich auch später auf ihren eigenen Schreibprozess auswirken wird.

Auch konzeptuell greift bei Merklinger (2011, S. 90 f.) die Skriptorin in den Text ein, indem sie zwar den Wortlaut des Kindes wiederholt, dabei aber Explizitformen verwendet, auf die Sprachangemessenheit achtet und grammatische Fehler korrigiert. Bei der Korrektur von lernersprachlichen Äußerungen waren wir im LAS-Projekt insgesamt jedoch zurückhaltender, um auch beim Schreibprozess des Kindes einen möglichst genauen Einblick in seinen eigenen Sprachstand zu bekommen. Wie bei der textstrukturellen Seite, ergaben sich jedoch auch im morphologisch-syntaktischen Bereich widersprüchliche Verhaltensanforderungen, die zu unterschiedlichen Lösungen führten.

Da die Aufgabenstellung insofern von Merklingers Design abwich, als die Erzählung des Films zu einem Ende geführt werden musste, war die Entscheidung, wann der Text fertig war, nicht allein dem Kind überlassen, sondern selbst ein Indikator für die Realisierung der Erzählstruktur. Den-

Wie im Abschlussbericht des Projekts LAS wurden hier für die Probanden pseudonymisierte Kürzel verwendet. Um einen Vergleich mit früheren Analysen dieser und anderer Texte der Kinder zu ermöglichen (Mehlem 2010, 2011), werden die jeweiligen Kodierungen einander zugeordnet: $\mathrm{KER}=\mathrm{Kemal}, \mathrm{OSM}=\mathrm{Ahmed}, \mathrm{FEH}=$ Fatma, $\mathrm{SEV}=$ Esra. 
noch kommen direkte Impulse, mit der Geschichte fortzufahren, in den Interaktionen nur sehr selten vor. Bei langen Pausen (z.B. FEH_T2_20) las z.B. die Skriptorin nochmals den bisher geschriebenen Text vor, um dem Kind den Anknüpfungspunkt zu erleichtern, oder stellte die Frage, und dann?‘ (FEH_T2_84).

Beide Mädchen thematisieren die verschiedenen Aspekte des Schreibprozesses eher selten. Unter dem Eindruck der Verlangsamung des Erzähltempos spricht FEH immer einzelne Sätze und zerlegt zweimal einen Satz in kleinere Äußerungseinheiten, während SEV mehrmals in den spontanen mündlichen Erzählfluss zurückfällt und - nach dem schreibbegleitenden Sprechen des Skriptors - nur je einmal das letzte Wort eines Satzes vorspricht bzw. wiederholt (SEV_T2_22/24). SEV thematisiert außerdem an einer Stelle einen vom Skriptor geschriebenen Buchstaben und fragt nach seinem Lautwert (SEV_T2_44-45). Dreimal korrigiert FEH im Nachhinein ihre Äußerungen, wobei sie das Vorhergehende zweimal explizit als fehlerhaft kennzeichnet (FEH_T2_59/82) bzw. einmal einen Teil der vorher gesprochenen Äußerung leicht verändert wiederholt. Dadurch signalisiert sie der Skriptorin, welche Teile ihres gesprochenen Textes keinen Eingang in die Schrift finden sollen. SEV problematisiert an keiner Stelle, ob der vom Skriptor artikulierte Text ihre Äußerungen tatsächlich vollständig wiedergibt.

\subsection{Interaktionen beim Schreibprozess zweier Schülerinnen}

Unmittelbar im Anschluss an das Diktat erhält das Kind die Aufgabe, die Erzählung selbst aufzuschreiben. Dabei steht der vom Skriptor festgehaltene Text nicht als direkte Quelle für das Kind zur Verfügung, wohl aber kann er vom Skriptor als Orientierungshilfe hinzugezogen werden. Das Design sah aber vor, dass möglichst das Kind allein auf allen Ebenen der Textproduktion eine neue Version des Textes erstellt.

Nur zwei der fünf Schreibszenen zeichnen sich durch eine intensive Interaktion zwischen schreibendem Kind und Feldassistenten aus. Die Interaktion dient gleichsam als Gerüst für den Schreibprozess, der mit ca. $25 \mathrm{Mi}-$ nuten die normale Spanne einer üblichen Schreibaufgabe im ersten Schuljahr deutlich übersteigt. Die Kinder befinden sich also in einer Situation, in der sie in besonderer Weise zum Schreiben eines längeren Textes herausgefordert werden.

Bei FEH fällt vor allem auf, dass sie mit äußerster Konzentration zu Werke geht. Der eigentliche Schreibvorgang wird kaum durch längere Denkpausen oder Nebeninteraktionen mit der Interviewerin unterbrochen. SEV dagegen ist immer wieder abgelenkt oder versucht, ihren Gesprächspartner von der Schreibaufgabe abzulenken. 


\subsubsection{Der mediale Schreibprozess als Gerüst}

Von den 18 satzförmigen Einheiten, die FEH schreibt, werden nur in sieben Fällen Planungsprobleme auf der Satzebene angesprochen. Dabei spielt sich zwischen beiden nach anfänglichen Unsicherheiten ein Verfahren ein, bei dem FEH die Interviewerin fragt, ob sie ein bestimmtes Wort schreiben soll. Da sich diese Frage nur beantworten lässt, wenn FEH's Schreibplan bekannt ist, fragt die Interviewerin zurück, was sie denn schreiben will, worauf FEH meist die entsprechende Erklärung in Form einer Wortgruppe oder eines ganzen Satzes gibt. Die Entstehung dieser kooperativen Strategie wird im Folgenden erläutert.

$\mathrm{Zu}$ Beginn ist zwischen beiden noch nicht geregelt, wie bei Schwierigkeiten im Schreibprozess vorgegangen werden könnte. Nach der Niederschrift der ersten Äußerung macht FEH eine lange Pause. Die Interviewerin versucht ihr zu helfen, indem sie auf ihre bereits vorliegende Verschriftung zurückgreift (FEH_T2_136-140), aber FEH hat wohl schon einen Plan für den nächsten Satz, der mit ,der ${ }^{6}$ beginnt (FEH_T2_141). Allerdings kann die Interviewerin ihr die Frage, ob sie nun ,der schreiben soll, ohne eine Verbalisierung des ganzen Schreibplans nicht beantworten. Sie liest daher den diktierten Text von FEH vor, der keinen entsprechenden Satz enthält (FEH_T2_144-149). Darauf insistiert FEH auf dem ,der', das sie dreimal wiederholt; ermutigt durch INT schreibt sie nun diesen Satz (,der hat was verloren? weiter, indem sie immer eine kleine Wortgruppe wiederholt, die auch das zu schreibende Wort enthält, und dann auf die Buchstabenebene wechselt.

Das zweite Planungsproblem auf Textebene entsteht nach dem zweiten Satz. FEH liest ihr ganzes bis dahin Geschriebenes vor und überlegt sehr lange, bevor INT die inhaltliche Frage nach dem Fortgang der Handlung zweimal stellt (FEH_T2_171/173) und das vorhergehende Ereignis nochmals benennt (172).

Transkriptauszug 1

\begin{tabular}{|l|l|l|l|}
\hline & & verbale Interaktion & Schreibung \\
\hline 171 & INT: & was passiert dann ? & \\
\hline 172 & INT: & dann hat das mädchen was verloren . & \\
\hline 173 & INT: & und dann ? & \\
\hline 174 & FEH: & kommt mädchen und jung ne ? & \\
\hline 175 & INT: & genau . & \\
\hline 176 & FEH: & kommt noch mal . & $<$ kommt $>$ \\
\hline 177 & FEH: & [kə] [?o] ... [tə] [ko:m:t]. & \\
\hline
\end{tabular}




\begin{tabular}{|l|l|l|l|}
\hline 178 & FEH: & mädchen . & $<$ Mecen $>$ \\
\hline 179 & FEH: & und . & $<$ und $>$ \\
\hline 180 & FEH: & und [?i:] ne ? & $<$ iung $>$ \\
\hline 181 & INT: & hmhm, genau, richtig . & \\
\hline
\end{tabular}

Nun spricht FEH selbst den ganzen dritten Satz (174) vor, der als Schreibplan durch INT bestätigt wird (175). Danach wechselt FEH von der Satzauf die Wortebene, indem sie sich die Wörter ,kommt' (176), ,Mädchen' (178) sowie ,und' (179) zurechtlegt. In den Äußerungen 177 und 180 befindet sie sich dagegen auf der segmentalen Ebene, auf der einzelne Lautsegmente herausgelöst und in Buchstaben übersetzt werden. Mithilfe von Rückfragen an die Interviewerin, die den größten Teil ihrer Interaktionen ausmachen, gelingt es FEH, in den basalen Schreibprozess eine klare Struktur zu bringen.

Gleichzeitig wird der konzeptuelle Planungsprozess stärker nach innen verlagert. Erst bei Satz 6 bittet sie INT wieder um Hilfe bei der Schreibung eines Wortes, womit aber der gesamte inhaltliche Schreibplan in Frage steht:

Transkriptauszug 2

\begin{tabular}{|l|l|l|}
\hline 207 & FEH: & muss ich wieder, das' schreiben ne ? (16:51) \\
\hline 208 & *IV2: & was willst du denn sagen ? \\
\hline 209 & FEH: & dann hat das jung das mädchen gegeben. \\
\hline 210 & *IV2: & hmhm@i. \\
\hline
\end{tabular}

Textauszug 1

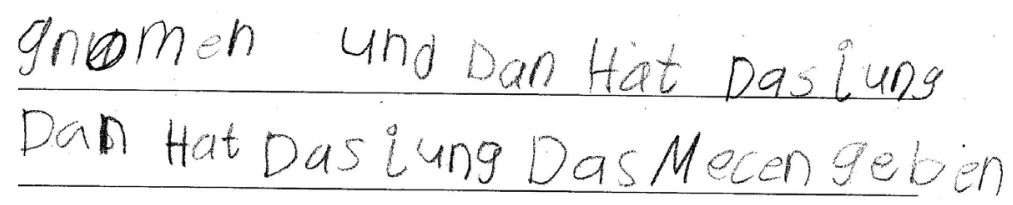

Es ist durch die Aufnahme nicht zweifelsfrei rekonstruierbar, an welcher Stelle im Schreibprozess FEH diese Frage stellt: bei dem später zu ,dan ${ }^{6}$ korrigierten ,das' am Anfang oder beim ,das' vor ,Mecen` in der Mitte der zweiten Zeile. Sie hat aber diesen Satz vor Augen, der tatsächlich zweimal das Wort ,das' enthält. Bei ihrer Frage an INT setzt FEH voraus, dass diese allein aufgrund der bereits geschriebenen Wörter diesen Schreibplan schon kennt. Erst durch deren Nachfrage wird der Schreibplan in Gestalt des Satzes expliziert, und nun kann INT die Korrektheit des zweiten ,das' bestätigen. In den vier verbleibenden übergreifenden Planungsstellen nimmt FEH immer ein fehlendes Wort oder eine konkrete Wortschreibung zum Anlass, 
um von der Interviewerin auch Hilfe bei der Textplanung zu bekommen, die nun aber zu Ende geführt wird, ohne dass jeweils der ganze geplante Satz vor der Niederschrift artikuliert wird.

\subsubsection{Der konzeptuelle Schreibprozess als Gerüst}

In der zweiten Schreibszene werden insofern die Rollen von Autor und Skriptor getauscht, als jetzt der Interviewpartner diktiert und das Kind weitgehend die Skriptorenrolle übernimmt. Von den zehn Sätzen, aus denen SEV's Text am Ende besteht, lassen sich sechs auf den Input des Interviewers zurückführen, einer entsteht gemeinsam aus den Redeanteilen beider, nur drei werden von SEV selbst formuliert (SEV_T2_141, 155-159; 191, 198; 201-215). Von den acht diktierten Sätzen des Interviewers, kennzeichnet dieser selbst einen als nicht relevant, ein weiterer wird von SEV nicht weiter beachtet. Drei der diktierten Sätze sind gegenüber dem Original nur auf wenige Worte reduziert, nur drei Sätze werden einigermaßen vollständig in den Text übernommen. Von den vier von ihr selbst formulierten Sätzen tauchen dagegen drei vollständig in der Verschriftung auf. Zwei der weggelassenen Sätze und ein stark reduzierter Satz sind übrigens Einleitungssätze von wörtlicher Rede (siehe unten).

Das Ineinandergreifen der Schreibprozesse auf den verschiedenen Ebenen soll wieder durch einen Ausschnitt veranschaulicht werden:

Transkriptauszug 3

\begin{tabular}{|l|l|l|}
\hline 147 & INT: & was hast denn da gerade geschrieben ? \\
\hline 148 & INT: & sach mal . \\
\hline 149 & INT: & lies doch noch mal . \\
\hline 150 & SEV: & [d] [u] . \\
\hline 151 & INT: & du . \\
\hline 152 & SEV: & [bə] [R:] [?i] [ts] [də] [?a] [z] . \\
\hline 153 & INT: & bringst ne ? \\
\hline 154 & SEV: & hmhm \\
\hline 155 & SEV: & [?i] [J] . \\
\hline 156 & INT: & ja genau . \\
\hline 157 & INT: & [S] . \\
\hline 158 & SEV: & [S]. \\
\hline 159 & SEV: & [də] [?a] [hə] [?i] [n] dahin . \\
\hline
\end{tabular}


SEV liest nicht den geschriebenen Text vor, sondern buchstabiert ihn. Das Angebot des Interviewers, der ein Wort und damit einen möglichen Schreibplan SEV's rekonstruiert (153), bestätigt sie zwar, führt die Äußerung aber nicht weiter, sondern fährt im schreibbegleitenden Lautieren fort (155). Nur das letzte Wort des Satzes wird nochmals als Ganzes gelesen (159). SEV operiert hier nicht auf drei, sondern überwiegend nur auf einer, der segmentalen (medialen) Ebene des Schreibprozesses, was sich auch darin zeigt, dass sie zwischen Wörtern keine Spatien setzt und so auch nicht mehr auf sie als bedeutungstragende Einheiten zurückgreifen kann:

Textauszug 2

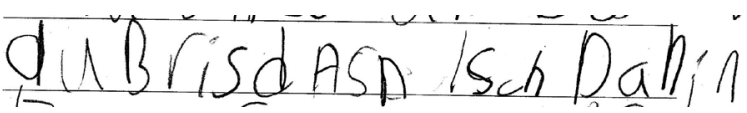

Dies erklärt nun auch die starke Übernahme der Textplanung durch den Interviewer, die - im Sinne der Verteilung unterschiedlicher Jobs durch unterschiedliche Sprecher bei der gemeinsamen interaktiven Hervorbringung der Erzählung - als partielle Übernahme der Autorfunktion verstanden werden kann. Als weiterer Faktor, der z.B. bei FEH nicht in Erscheinung trat, sind die zahleichen Versuche SEV's, aus der Schreibsituation auszusteigen, zu berücksichtigen. Von ihren ca. 80 Äußerungen während des Schreibens zielen allein 14 darauf, dass sie ein Wort nicht schreiben kann (220, 283), oder dass der Interviewer schreiben soll $(224,287)$ bzw. über etwas anderes, nämlich die bevorstehende Kirmes $(263,269)$, zu reden. Neben den zahlreichen Ermunterungen, mit denen der Interviewer SEV bei der Stange hält, stellt vielleicht auch die teilweise Übernahme der Diktierfunktion einen Versuch dar, der Schreiberin nach dem Übergabeprinzip zunächst nur die lokale/mediale Textgestaltung zu übertragen. Diese Strategie erweist sich insofern als erfolgreich, als SEV tatsächlich - im Unterschied zur Schreiberin ALE (siehe unten) - auch im Geschriebenen die Geschichte abschließt.

\section{Literate Strukturen am Beispiel von Nominalphrasen}

Für die Analyse des Verhältnisses orater und literater Strukturen wurde im Anschluss an Maas (2008, 2010) ein Verfahren entwickelt, bei dem die formalen sprachlichen Merkmale des Textes und auch einige funktionale Aspekte berücksichtigt wurden. Im Folgenden werden - am Beispiel der Nominalphrasen - die Ergebnisse dieser Analyse jeweils für die diktierten und die geschriebenen Texte vorgestellt. ${ }^{3}$

Das Verfahren wird derzeit noch modifiziert, um auch sprachübergreifende Vergleiche mit dem Türkischen und dem Kurdischen zu ermöglichen. Insofern sind kleinere Abweichungen zu anderen Formen der Auszählung in anderen Publikationen aus dem Projektkontext möglich. 
Gegenüber rein quantitativen Maßen wie der lexikalischen Dichte (also dem Verhältnis von Funktions- und Inhaltswörtern, vgl. Laufer/Nation 1995; O'Loughlin 1995; Read 2000) und der lexikalischen Diversität (type-tokenratio), die in neueren Studien zur Entwicklung akademischer Sprache bei Vorschul- und Grundschulkindern eine große Rolle spielen (Schleppegrell 2004; Leseman et al. 2007, 2009), hat die orat-literat-Analyse den Vorteil, dass sie auch bei relativ geringen Textmengen Aussagen zum Grad der Literalität eines Textes ermöglicht.

Ausgehend vom Bühlerschen Begriff des Symbolfelds (Bühler 1934; Maas 2010, S. 7 ff.) kann literate Sprache zunächst so verstanden werden, dass mithilfe von lexikalischen Wortformen auf Sachverhalte in der Welt referiert wird. Im Bereich der nominalen Kategorien wären also lexikalische NPs zunächst Kandidaten für literate Strukturen im Gegensatz zu pronominal oder nullmarkierten Argumentpositionen. Aber schon der Blick auf einfache Texte zeigt, dass ein solches Vorgehen die Leistungen der Anapher und der Koordinatenreduktion bei der Generierung kontextentbundener Satzstrukturen ausklammert. Diese müssen deshalb in die Analyse einbezogen werden. Ein weiterer Faktor ist die zentrale Funktion des Satzes (im Gegensatz zur Äußerung als kommunikativer Einheit einerseits und zur semantisch bestimmten Proposition andererseits), die Maas als Nexusfeld eines Prädikats (ebd., S. 82) definiert. Damit bilden alle nominalen Argumentpositionen eines Prädikats, und nicht nur die realisierten, einen weiteren unverzichtbaren Bezugspunkt. Die schon auf der Ebene der Diktier- und Schreibinteraktionen als für den Erfolg des Schreibens ausschlaggebende Kategorie des Satzes erhält damit auch für die orat-literat-Analyse eine besondere Relevanz.

Aus der bisherigen Darstellung ergibt sich eine skalare Struktur der Kategorie literat (ebd., S. 92 ff.). Für die folgende Analyse wurden vier Zwischenstufen gebildet, mit deren Hilfe die auf einem Kontinuum vom oraten zum literaten Pol auftretenden unterschiedlichen Ausprägungen von Nominalphrasen kodiert wurden. Dabei wird im Sinne der Entfaltung der Darstellungsfunktion der Sprache als erstes Kriterium der literaten Textstruktur die lexikalische Explizitheit gewählt, ergänzt durch Aspekte der Textkohäsion mithilfe unterschiedlicher pronominaler Formen sowie Markierungen der Referenteneinführung und Wiederaufnahme.

1) Am oraten Pol sind zunächst nicht lexikalische Nominalphrasen, die zur Referenteneinführung dienen, angesiedelt, ebenso direkte Verweise auf den Film sowie Referenzen auf den Sprecher oder den Hörer, bloBe Markierungen am Verb, die nicht in Kontexten von Koordinatenreduktion stehen, schließlich Tilgungen von Nominalphrasen in (aus der Perspektive eines vollständigen Satzes) obligatorischen Kontexten. 
2) Als neutral gelten Referenteneinführungen mit Indefinitpronomen oder Quantifizierer ,jemand', ,zwei', pronominale Wiederaufnahmen mit dem Demonstrativum ,der', unmarkierte lexikalische Nominalphrasen, die in Kontexten der Referenteneinführung stehen (oder indefinit markierte Nominalphrasen, die nicht in Kontexten der Referenteneinführung stehen).

3) Als literat gelten lexikalische Nominalphrasen, wenn sie in Kontexten der Einführung indefinit markiert sind, sowie definit markierte oder nullmarkierte Nominalphrasen in allen übrigen Kontexten; darüber hinaus aber auch Personalpronomina der ersten und zweiten Person sowie Wiederaufnahmen durch das anaphorische Pronomen ,er' (bzw. ,der ${ }^{`}$ in Sätzen unmittelbar nach der Referenteneinführung); schließlich Indefinitpronomina, die auf einen Kontext verweisen, in dem keine definite Referenz möglich oder nötig ist.

4) Als fortgeschritten literat gelten durch Adjektivattribut, Genetivattribut oder Relativsatz erweiterte Nominalphrasen, wobei hier nicht zwischen Links- und Rechtserweiterung der NP unterschieden wird (vgl. Ravid et al. 2002; Ravid 2006).

Bei den Kategorien der orat-literat-Analyse sollen zunächst lernersprachliche Phänomene kategorisch ausgeklammert werden. So werden im Falle der Nominalflexion Normabweichungen bei den Genus- und Kasusmarkierungen der Artikel, Pronomina und Adjektive nicht berücksichtigt. Da sich aber die Definitheitsmarkierung mit der Textstruktur überlagert, mussten zumindest Fälle der nicht-definiten Referenteneinführung berücksichtigt werden. Auch fehlende pronominale Subjektmarkierungen, die auch in oraten Texten von L1-Sprechern auftreten könnten, und fehlende Objektmarkierungen wurden daher in die orat-literat-Analyse einbezogen, da sie auch Lücken in der Argumentstruktur des Satzes darstellen, die im interaktiven Kontext vom Hörer leichter gefüllt werden können.

An der folgenden Gegenüberstellung der beiden Textanfänge von FEH soll das Verfahren der orat-literat-Analyse demonstriert werden:

Transkriptauszug 4

\begin{tabular}{|l|l|l|l|}
\hline & FEH D3 (diktiert) & & FEH D4 (schriftlich) \\
\hline 01 & da kommt ne frau . (+) & 01 & Da kommt Mecen (0). \\
\hline 02 & der hatte was . & & \\
\hline 03 & $\begin{array}{l}\text { der (+) hat was (0) } \\
\text { verloren . }\end{array}$ & 02 & Der (+) Hat Was (0) Valon. \\
\hline 04 & $\begin{array}{l}\text { und ähm da kommt mädchen } \\
(0) \text { und junge (0). }\end{array}$ & 03 & kommt Mecen (0) und jung. (0) \\
\hline
\end{tabular}




\begin{tabular}{|c|c|c|c|}
\hline & FEH D3 (diktiert) & & FEH D4 (schriftlich) \\
\hline 05 & $\begin{array}{l}\text { ham die (+) das (0) } \\
\text { gesehen }\end{array}$ & 04 & Der (0) Habn Das (0)gesen. \\
\hline 06 & $\begin{array}{l}\text { und dann ähm@i erstmal } \\
\text { hat die jung (+) } \\
\text { gesehen. }\end{array}$ & & \\
\hline 07 & $\begin{array}{l}\text { und dann hat die mädchen } \\
(+) \text { gesehen } \text {. }\end{array}$ & & \\
\hline 08 & $\begin{array}{l}\text { ähm und der und der } \\
\text { mädchen (+) und jung (0) } \\
\text { haben reingeguckt. }\end{array}$ & & \\
\hline 09 & $\begin{array}{l}\text { äh und dann habm (-) das } \\
\text { gesehen. }\end{array}$ & & \\
\hline 13 & $\begin{array}{l}\text { erstmal hat die jungen } \\
(+) \quad(-) \text { genommen }\end{array}$ & 05 & $\begin{array}{l}\text { und Dan Hat Das jung }(+)(-) \\
\text { gnomen. }\end{array}$ \\
\hline 14 & und dann $+\ldots$ & 06 & und Dan Hat Das jung $+/ /$. \\
\hline 15 & $\begin{array}{l}\text { hat } n \text { mädchen (0) was } \\
(0) \text { gesagt. }\end{array}$ & & \\
\hline 16 & $\begin{array}{l}\text { und dann hat die ähm } \\
\text { junge (+) das geld (+) } \\
\text { in die mädchens (+) } \\
\text { gegeben. }\end{array}$ & 07 & $\begin{array}{l}\text { Dan Hat Das jung }(+)(-) \text { Das } \\
\text { Mecen }(+) \text { geben. }\end{array}$ \\
\hline
\end{tabular}

Die fehlenden Artikel bei den Referenteneinführungen in D3-4 sowie D4-1 und D4-3 deuten hier auch auf ein lernersprachliches Problem hin, allerdings ist im ersten mündlichen Satz zumindest ein reduzierter unbestimmter Artikel vorhanden. Diese wirken sich aber auch auf die mangelnde Kontextentbundenheit des Textes aus. Dagegen spielen die abweichenden Genusmarkierungen für die Verständlichkeit des Textes keine Rolle bis auf D4-4, wo zwischen der Singularform ,der ${ }^{\varsigma}$ und der Pluralform des Verbs Inkongruenz besteht, die sich eventuell durch einen Bruch im Schreibplan erklären lässt. Das Fehlen von im Deutschen obligatorischen Subjekt- (D3-9) oder Objektpositionen (D4-5 und D4-7) wirkt sich ebenfalls auf die Explizitheit des Textes aus. Die Verteilungen entlang des orat-literat-Kontinuums sind damit mündlich 1:6:12 und schriftlich 2:6:4, ergeben also einen deutlich weniger literaten geschriebenen Text.

In der folgenden Übersicht sind die absoluten Zahlen in den deutschen Texten der fünf Kinder jeweils getrennt für mündlich (D3) und schriftlich (D4) dargestellt. Zur Berechnung des Literalitätsindex wurden zunächst die vier literalen Kategorien mithilfe der Faktoren 0,1,2 und 3 gewichtet, dann addiert und schließlich durch die Summe aller Nominalphrasen dividiert. 


\begin{tabular}{|l|l|c|c|c|c|c|c|}
\hline \multicolumn{2}{|c|}{} & lit - & neutral 0 & lit + & lit ++ & gesamt & Index-lit \\
\hline \multirow{2}{*}{ FEH } & D3 & 4 & 13 & 16 & 0 & 33 & 1,36 \\
\cline { 2 - 8 } & D4 & 6 & 9 & 13 & 0 & 28 & 1,25 \\
\hline \multirow{3}{*}{ OSM } & D3 & 0 & 6 & 9 & 0 & 15 & 1,60 \\
\cline { 2 - 8 } & D4 & 4 & 3 & 4 & 0 & 11 & 1,00 \\
\hline \multirow{3}{*}{ KER } & D3 & 1 & 15 & 12 & 1 & 29 & 1,45 \\
\cline { 2 - 9 } & D4 & 0 & 3 & 13 & 0 & 16 & 1,81 \\
\hline \multirow{3}{*}{ ALE } & D3 & 0 & 16 & 38 & 0 & 54 & 1,70 \\
\cline { 2 - 8 } & D4 & 2 & 2 & 13 & 0 & 17 & 1,65 \\
\hline \multirow{2}{*}{ SEV } & D3 & 0 & 12 & 14 & 1 & 27 & 1,59 \\
\cline { 2 - 8 } & D4 & 2 & 2 & 10 & 1 & 15 & 1,67 \\
\hline
\end{tabular}

Tab. 1: Verteilung der Nominalphrasen und Literalitätsindex in den diktierten (D3) und geschriebenen (D4) Erzählungen

Die Tabelle zeigt zunächst große quantitative Unterschiede zwischen den gesprochenen und geschriebenen Texten bis auf FEH, deren beide Texte etwa gleich viele Nominalphrasen enthalten. Demgegenüber scheinen die Verschiebungen des Literalitätsindex zwischen mündlich und schriftlich bei den drei Mädchen nur gering: Bei FEH und ALE nehmen literate Markierungen etwas ab, bei SEV dagegen zu. Aus diesem Bild fallen einerseits OSM mit einer starken Abnahme literater Strukturen im Schriftlichen und KER mit einer fast ebenso großen Zunahme. Auffällig ist die Zunahme besonders orater Strukturen in allen schriftlichen Texten bis auf KER's. Bei SEV ist die starke Unterstützung durch den Interviewer in Rechnung zu stellen, weshalb dieser Text in der LAS-Auswertung nicht berücksichtigt wurde. Die Erwartung einer Zunahme literater Indikatoren durch den Medienwechsel wird also nur durch KER bestätigt. Dies entspricht aber zahlreichen Befunden aus Analysen früher Verschriftungen, in denen die Explizitheit des mündlichen Textes unterschritten wurde (Maas/Mehlem 2003).

\section{Narrative Kompetenzen am Beispiel globaler Elemente der Erzählstruktur}

\subsection{Die Erzählung als hierarchische Struktur}

Die orat-literat-Analyse erlaubt keine Aussagen über die semantische Kohärenz eines Textes und seine besonderen Merkmale mit Bezug auf eine bestimmte Textsorte. Die einzelnen Elemente werden nur in ihrer globalen Verteilung erfasst, durch die Unterscheidung von Referenteneinführung und Wiederaufnahme werden nur ansatzweise unterschiedliche Funktionen im 
Text einbezogen. Lexeme und Pronomina als Mittel der Kohäsion können zwar identifiziert werden, aber nicht ihr Beitrag zur Textkohärenz. Deshalb wird nun im zweiten Schritt eine auf die Kohärenz bezogene Perspektive eingenommen.

Bei der Analyse der narrativen Struktur der Texte wird auf ein eher traditionelles Verfahren der Rekonstruktion der ,kognitiven Geschichte (Quasthoff 1980; Hausendorf/Quasthoff 2005) bzw. der Musterrealisierung (Rehbein 2007) zurückgegriffen. Dabei geht es zunächst um die Identifizierung relevanter Ereignisknoten, die ein hierarchisches Gefüge bilden. In Anknüpfung an und Weiterentwicklung des Bielefelder Geschichtenschemas (Boueke et al. 1995; Wolf/Boueke/Schülein 2007) wurde speziell für den Fall des Stummfilms aufbauend auf Blaschitz (2010, S. 105) ein Analyseraster entwickelt, das zunächst bestimmte Inhaltselemente innerhalb von Szenen oder Episoden identifiziert.

\begin{tabular}{|c|c|c|c|c|c|}
\hline \multirow{4}{*}{$\begin{array}{l}\text { Szene } \\
1\end{array}$} & 1 & Protagonistin A & $\begin{array}{l}\text { Szene } \\
5\end{array}$ & (9) & $\begin{array}{l}\text { Besitzersuche Teil } \\
\text { 1: Protagonistin D } \\
\text { (Erfolglose Suche) }\end{array}$ \\
\hline & $1 \mathrm{a}$ & Weg Büro & & $7 \mathrm{a}$ & $\begin{array}{l}\text { Annäherung an } \\
\text { Protagonistin D }\end{array}$ \\
\hline & 2 & Verlust des Kuverts & & $7 \mathrm{~b}$ & $\begin{array}{l}\text { Anliegensschilderung an } \\
\text { Protagonistin D }\end{array}$ \\
\hline & $2 \mathrm{a}$ & Nicht-Bemerken Verlust & & $7 \mathrm{c}$ & $\begin{array}{l}\text { Verneinung Protagonis- } \\
\text { tin D }\end{array}$ \\
\hline \multirow{3}{*}{$\begin{array}{l}\text { Szene } \\
3\end{array}$} & 3 & Protagonisten B und C & & 8 & $\begin{array}{l}\text { Verweis auf A durch } \\
\text { Protagonistin D }\end{array}$ \\
\hline & 4 & Finden des Kuverts & & 9 & $\begin{array}{l}\text { Besitzersuche Teil 2: Suche } \\
\text { nach A }\end{array}$ \\
\hline & $4 a$ & Inhalt des Kuverts: Geld & $\begin{array}{l}\text { Szene } \\
6\end{array}$ & 10 & $\begin{array}{l}\text { Annäherung an } \\
\text { Protagonistin A, gefunden“ }\end{array}$ \\
\hline \multirow{7}{*}{$\begin{array}{l}\text { Szene } \\
4\end{array}$} & $5 \mathrm{a}$ & Konflik:t: Behalten-Wollen & & $10 \mathrm{a}$ & $\begin{array}{l}\text { Anliegensschilderung an } \\
\text { Protagonistin A }\end{array}$ \\
\hline & $5 b$ & $\begin{array}{l}\text { An sich-Nehmen Kuvert: } \\
\text { Protagonist C }\end{array}$ & & $10 \mathrm{~b}$ & $\begin{array}{l}\text { Fühlen in Hosentasche: } \\
\text { kein Geld }\end{array}$ \\
\hline & $5 c$ & Konflikt: Zurückgeben-Wollen & & $11 \mathrm{a}$ & Freude \\
\hline & $5 \mathrm{~d}$ & Kritik B an C & & $11 b$ & Dank \\
\hline & $6 \mathrm{a}$ & $\begin{array}{l}\text { Überlegung weiteres } \\
\text { Verfahren }\end{array}$ & & $11 \mathrm{c}$ & $\begin{array}{l}\text { Übergabe Kuvert und } \\
\text { Geld }\end{array}$ \\
\hline & $6 \mathrm{~b}$ & Nachgeben von $\mathrm{C}$ & & $11 d$ & Bestätigung: mein Geld! \\
\hline & $6 c$ & $\begin{array}{l}\text { Lösung Konflikt: } \\
\text { Besitzersuche }\end{array}$ & & & \\
\hline
\end{tabular}

Tab. 2: Szenengliederung des Films und Zuordnung von Inhaltselementen 
Aus dieser Tabelle lässt sich noch keine hierarchische Struktur der Inhaltselemente ableiten. Diese kann mithilfe eines zuerst im Rahmen der story grammar entwickelten Baumdiagramms veranschaulicht werden: Für die Realisierung der Geschichte des Films ist es entscheidend, dass die Elemente 1-4 und 9-10 realisiert werden: Die Einführung der Protagonistin A installiert gleichzeitig einen Agentenplan (Weg ins Büro), der durch den zunächst unbemerkten Verlust des Kuverts gebrochen wird (Komplikation). Das Auftreten der beiden Finder (2. Episode = Szene 3) stellt noch keine Auflösung des Planbruchs dar, da nun das Kuvert erst noch zu seiner Besitzerin gebracht werden muss. Die dritte Episode (Konflikt) markiert insofern den ersten Schritt zur Auflösung, als die beiden Finder sich darauf einigen, nach dem Besitzer zu suchen. Über die zunächst erfolglose Suche als retardierenden Moment (4. Episode, Szene 5) gelangt der Umschlag in Episode 5 wieder in die Hände der Protagonistin A.

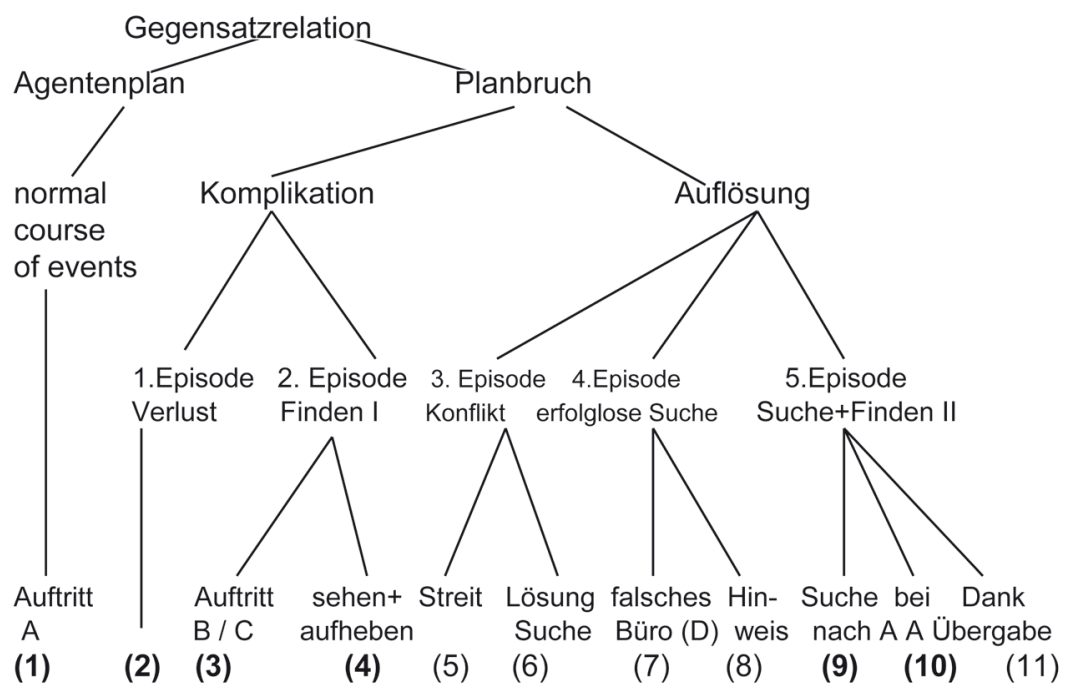

Abb. 1: Baumstruktur der Stummfilmerzählung (nach Quasthoff 1980, S. 105)

\subsection{Globale Erzählstrukturen in den Schülertexten}

Im Gegensatz zu älteren Schülern haben die Erstklässler unseres Projekts von sich aus den Konflikt in der Regel nicht oder nur in einer Weise thematisiert, der den genauen Hintergrund des Streits als moralisches Dilemma nicht erkennen ließ. Dagegen wurde von einigen Schülern die erfolglose Suche aufgrund des gut sichtbaren Raumwechsels und des Auftretens der Protagonistin D angesprochen, bevor die Auflösung in der 5. Episode thematisiert wurde. Ein Index der narrativen Struktur wurde gebildet, in dem jedes sprachlich realisierte Inhaltselement aufgrund seiner Stellung in der 
Relationsstruktur unterschiedlich gewichtet wurde: Die bereits erwähnten Hauptknoten wurden mit fünf Punkten gewichtet, das Nichtbemerken des Verlusts und Freude und Dank bei der Übergabe als Komplikation und Auflösung verstärkende Elemente der ,psychologischen Nähe' (Boueke et al. 1995) mit vier Punkten, ein Bezug zum Konflikt der dritten Episode mit drei, die vierte Episode mit zwei und jedes weitere, auch unspezifische Inhaltselement mit nur einem Punkt. Bei den Hauptknoten wurde darüber hinaus berücksichtigt, in welchem Umfang das Inhaltselement aufgrund der sprachlichen Form seiner Realisierung identifizierbar ist. Dabei wurde zwischen vollständig identifizierbar (5 Punkte), nur teilweise identifizierbar (3 Punkte) und nicht identifizierbar (1 Punkt) unterschieden.

Hieraus ergibt sich folgende Verteilung für die bereits untersuchten zehn Texte (siehe Tab. 3). Dabei bezieht sich die erste Zahl ausschließlich auf den Knoten der Wiederaufnahme von A, die zweite auf die Realisierung der Hauptknoten, die dritte auf alle Inhaltselemente.

\begin{tabular}{|c|c|c|c|c|}
\hline \multicolumn{2}{|c|}{} & \multicolumn{3}{|c|}{ narrativer Index } \\
\cline { 3 - 5 } \multicolumn{2}{|c|}{} & $\begin{array}{l}\text { Wiederauf- } \\
\text { nahme der } \\
\text { Protago- } \\
\text { nistin A }\end{array}$ & $\begin{array}{c}\text { Haupt- } \\
\text { knoten }\end{array}$ & gesamt \\
\hline \multirow{2}{*}{ FEH } & D3 & 6 & 26 & 37 \\
\cline { 2 - 5 } & D4 & 10 & 30 & 44 \\
\hline \multirow{2}{*}{ OSM } & D3 & 3 & 22 & 30 \\
\cline { 2 - 5 } & D4 & 2 & 20 & 22 \\
\hline \multirow{3}{*}{ KER } & D3 & 10 & 30 & 46 \\
\cline { 2 - 5 } & D4 & 8 & 28 & 37 \\
\hline \multirow{2}{*}{ ALE } & D3 & 10 & 30 & 59 \\
\cline { 2 - 5 } & D4 & 0 & 20 & 32 \\
\hline \multirow{2}{*}{ SEV } & D3 & 10 & 26 & 47 \\
\cline { 2 - 5 } & D4 & 10 & 26 & 40 \\
\hline
\end{tabular}

Tab. 3: Index der narrativen Struktur

Interessanterweise treten bei allen Schülern, wenn auch auf unterschiedlichem Ausgangsniveau, bei der schriftlichen Textversion z.T. deutliche Rückschritte gegenüber dem Mündlichen auf. Die einzige Ausnahme ist FEH, die sich in der schriftlichen Version steigert. Besonders gut lässt sich die unterschiedliche Qualität der narrativen Struktur an der Wiedereinführung der Protagonistin A erkennen, die im Schlussteil der Erzählung als diejenige identifiziert werden muss, die am Anfang den Umschlag verloren hat. Drei 
der fünf Kinder realisieren diese Aufgabe angemessen im Mündlichen, zwei im Schriftlichen. OSM gelingt es in beiden deutschen Versionen nicht, die Pointe zu erfassen. ALE bricht die schriftliche Erzählung kurz vor der Schlussepisode ab. An den unterschiedlichen Formen der sprachlichen Realisierung dieser Schlüsselstelle sollen nicht nur die unterschiedlichen Grade der semantisch-narrativen Realisierung verdeutlicht, sondern auch gegenüber der orat-literat-Analyse abgegrenzt werden.

\subsection{Syntaktische Form und narrative Funktion der Wiederaufnahme}

Die Wiedereinführung der Protagonistin A kann mit sehr verschiedenen sprachlichen Mitteln realisiert werden. Im LAS-Korpus finden sich folgende Varianten:

1) restriktiver Relativsatz

„Wo ist diese Frau, das das Geld verloren hat.“ (SEV D3, D4)

2) nicht-restriktiver Relativsatz „sie haben gefunden, den das gehört“" (KER D3)

3) indirekter Fragesatz „da wollte das Medchen fragen, wem das Geld gehört.“ (KER D4) „sie haben gefragt ob die gehört.“ (FEH D4)

4) definite NP mit direkter Rede „und dann hat gesagt die frau. ja, das gehört mir.“(FEH D3, 26-27; D4)

Neben diesen Formen treten auch sprachliche Mittel auf, die keine eindeutige Identifizierung mehr zulassen:

5) Deixis

,(14) gehen sie nach dem 15 ähm mädchen 16 die sagt 17 woanders ist das" (OSM D3)

6) Indefinitpronomen

„Dann sind sie zu einer anderen gegangen.“ (OSM D4)

Unter dem Gesichtspunkt der narrativen Struktur (Realisierung der Auflösung) sind alle Strukturen gleich gut, solange sie dem Hörer die Identifizierung der ersten Protagonistin und den Nachvollzug der ,Heimkehr ${ }^{6}$ des verlorenen Umschlags ermöglichen. Daher konnten die Lösungen 1-4 unbeschadet ihrer unterschiedlichen syntaktischen Struktur als vollständige Realisierung des narrativen Knotens gewertet werden, während nur die Hypotaxen 1-3 auch auf dem orat-literat-Kontinuum komplexe Strukturen darstellen.

Für die Verständlichkeit der Erzählung sind allerdings solche Formulierungen besser, die den Auflösungsknoten nicht nur einmal, sondern mehrfach thematisieren, und so eine Spannung vom Beginn bis zum Ende der Suche aufbauen. In der folgenden Tabelle sind die Texte nach diesen unterschiedlichen Realisierungen eingeordnet. 


\begin{tabular}{|c|c|c|c|c|}
\hline $\begin{array}{l}\text { keine } \\
\text { Identifizierung } \\
\text { möglich }\end{array}$ & $\begin{array}{l}\text { einfache } \\
\text { Realisierung } \\
\text { (Knoten 10) }\end{array}$ & $\begin{array}{l}\text { einfache } \\
\text { vollständige } \\
\text { und weitere } \\
\text { unvollständige } \\
\text { Realisierung }\end{array}$ & $\begin{array}{l}\text { doppelte } \\
\text { Realisierung } \\
\text { (Knoten 6/7 } \\
\text { und 10) }\end{array}$ & $\begin{array}{l}\text { dreifache } \\
\text { Realisierung } \\
\text { (Knoten } 7, \\
9 \text { und } 10)\end{array}$ \\
\hline $\begin{array}{l}\text { OSM D3 } \\
\text { OSM D4 } \\
\text { ALE D4 }\end{array}$ & FEH D3 & $\begin{array}{l}\text { ALE D3 } \\
\text { KER D4 }\end{array}$ & $\begin{array}{l}\text { SEV D3 } \\
\text { SEV D4 } \\
\text { KER D3 }\end{array}$ & FEH D4 \\
\hline
\end{tabular}

Tab. 4: Vorankündigung und Gestaltung des Auflösungsknotens

Interessanterweise sind es neben dem mündlichen Text von KER die beiden schriftlichen Texte von SEV und FEH, denen eine bessere Strukturierung des Auflösungsknotens gelingt. Der Relativsatz, den SEV in der mündlichen Version verwendet, gehört zu den wenigen Sätzen, die sie auch bei der Verschriftung selbst formuliert. Beide Schreiberinnen können also die Interaktion teilweise auch zur narrativen Ausgestaltung beim Schreibprozess nutzen.

\subsection{Formen der Figurenrede}

Die im vorhergehenden Abschnitt untersuchten unterschiedlichen Lösungen bei der Wiederaufnahme verweisen auf einen weiteren Aspekt, in dem literate und narrative Strukturen auseinander treten: den Gebrauch der Figurenrede. In ihrer narrativen Funktion kommt der Figurenrede vor allem im szenischen Erzählen eine Schlüsselstellung zu: Sie thematisiert das Geschehen aus der Perspektive einer Figur und erlaubt die Veranschaulichung des Gegenhandelns der Akteure durch einen Wortwechsel. Auch können die Handlungsziele der Akteure durch eine Rede wiedergegeben werden, ohne dass Modalkonstruktionen verwendet werden müssen. Bei der Verteilung der direkten Rede in den Texten werden zunächst Fälle uneingeleiteter und eingeleiteter Rede unterschieden.

\begin{tabular}{|c|c|c|c|c|c|c|c|c|c|c|c|}
\hline & \multicolumn{2}{|c|}{ KER } & \multicolumn{2}{|c|}{ OSM } & \multicolumn{2}{|c|}{$\mathrm{FEH}$} & \multicolumn{2}{|c|}{ SEV } & \multicolumn{2}{|c|}{ ALE } \\
\hline & & D3 & D4 & D3 & D4 & D3 & D4 & D3 & D4 & D3 & D4 \\
\hline \multirow{2}{*}{$\begin{array}{l}\text { direkte } \\
\text { Rede }\end{array}$} & + eingebettet & & & 1 & & 2 & 3 & 3 & 1 & 6 & \\
\hline & - eingebettet & & & & & & & 2 & 2 & 2 & 2 \\
\hline \multirow{2}{*}{$\begin{array}{c}\text { indirekte } \\
\text { Rede }\end{array}$} & + eingebettet & 2 & 1 & & & & 2 & & & & \\
\hline & - eingebettet & & & & & & & & & 1 & \\
\hline \multicolumn{2}{|c|}{ gesamt } & 2 & 1 & 1 & 0 & 2 & 5 & 5 & 3 & 9 & 2 \\
\hline
\end{tabular}

Tab. 5: Formen der Figurenrede 
Die Tabelle zeigt einen deutlichen Unterschied zwischen zwei Gruppen von Texten: Nur Texte der drei Mädchen weisen relativ hohe Anteile wörtlicher bzw. indirekter Rede auf, wobei bei ALE und SEV jeweils die mündlichen, bei FEH aber der schriftliche Text in diesem Bereich (>4) liegen. Dies sind auch die Texte mit dem höchsten Grad narrativer Entfaltung; in dieser Hinsicht stellt nur KER's mündlicher Text eine Ausnahme dar, der aber als einziger eine Art von berichteter Rede enthält.

Nicht eingebettete direkte Rede tritt besonders häufig in den beiden schriftlichen Texten von ALE und SEV und außerdem in SEV's mündlichem Text auf. In den mündlichen Texten lässt sich ein Fehlen der Einleitungssätze z.T. dadurch erklären, dass zwischen zwei Akteuren eine schnelle Wechselrede stattfindet, die das jeweilige Gegenhandeln besonders hervorhebt. Dies gilt für die beiden mündlichen Texte von SEV und ALE, in denen z.T. ein identischer Wortlaut wiederholt wird. Mit Rehbein (2007) lassen sich hier Spuren der in der türkischen Erzählkultur beliebten schnellen Wechselrede erkennen, und in der Tat findet sich diese auch besonders ausgeprägt in einem der türkischen Texte von SEV.

Transkriptauszug 5

SEV: Iki tane kadınla ve bir tane adam geldi.

Mit zwei Frauen... und ein Mann kam

IV : hmhm .

08

SEV: söyle diler +"bu bizim değil" - "bizim” .

Sie sagten: "Das gehört uns nicht." -

"Doch, uns!"

IV : hmhm .

RAH : +"bizim değil" - "bizim" .
"gehört uns nicht." - "Doch, uns!"

IV: hmhm .

In Bezug auf die beiden Kontinua orat-literat und narrativ entfaltet würden derartige Strukturen einerseits dem oraten, andererseits dem narrativ entfalteten Pol zustreben.

Dies verhält sich zunächst konträr zu den Anforderungen der Schriftlichkeit, in der ja eine Rahmung des ,kommunikativen Fensters' (Pohl 2004, S. 5) gerade nicht durch Prosodie erfolgen kann. Eine aus der Interaktionssituation z.T. rekonstruierbare Erklärung kann darin gesehen werden, dass durch die Zwänge der medialen Bewältigung eine Konzentration auf den für die Handlung am wenigsten verzichtbaren Sachverhalt stattfindet. 
Alle Einleitungssätze der direkten bzw. indirekten Rede bis auf einen sind vorangestellt. Hier ist die Erklärung Pohls heranzuziehen, der von einem ikonischen Verhältnis zwischen Konstatieren der Rede und der Wiedergabe des Redeinhalts ausgeht (Pohl 2004, S. 8). Die einzige Abweichung von diesem Prinzip bei FEH lässt sich dadurch erklären, dass im Schreibprozess der Einleitungssatz unvollendet bleibt und daher eine zweite Fassung nochmals nachgeschoben wird. Dass andererseits die Ausgestaltung des Redebegleitsatzes zwar literater Explizitheit, aber nicht zwingend narrativer Gestaltung dient, bestätigt Pohls Analyse insofern, als professionelle Erzähler die größere semantische Komplexität der Begleitsätze wieder abbauen (ebd., S. 10 f.).

\subsection{Narrative und literale Indikatoren im Überblick}

Die Grafik (Abb. 2) bestätigt zunächst den grundsätzlichen Trend einer relativ starken Übereinstimmung literaler und narrativer Indikatoren. Die mündlichen und schriftlichen Texte der fünf Schüler lassen sich in einem Cluster anordnen, das bei steigender narrativer Komplexität auch mit einer größeren Explizitheit der Nominalphrasen einhergeht. Dieser allgemeine Trend wird aber von den unterschiedlichen Schülern auf sehr verschiedene Weise realisiert. In den schriftlichen Texten wird tendenziell eine größere Explizitheit, in den mündlichen eine stärkere narrative Entfaltung erreicht. Dies entspricht der Ausgangshypothese, dass in der frühen Phase des Schreibprozesses die Absorption durch das Medium eine größere sprachliche Entfaltung stärker behindert als die explizitere Markierung der Nominalphrasen. Allerdings folgen nur drei Schüler eindeutig diesem Trend. FEH nutzt gerade den Schreibprozess, um die narrative Komplexität zu erhöhen, während OSM in seiner Verschriftung sowohl literal wie narrativ hinter das im Mündlichen Erreichte zurückfällt.

\section{Zusammenfassung}

Ziel der hier vorgestellten Analyse war es, den Gebrauch des Deutschen in gesprochener und geschriebener Sprache durch Erstklässler mit türkischer Erstsprache im Spannungsverhältnis narrativer und literater Indikatoren darzustellen. Ausgehend von einem Untersuchungsdesign, das die Erfahrung des Diktierens für die Auseinandersetzung mit medialer und konzeptueller Schriftlichkeit nutzt und die verbale Interaktion mit dem Interviewer ins Schreiben hinein verlängert, konnte gezeigt werden, in welchem Maße die Strukturierung des Textes auch auf der medialen Ebene zur inhaltlichen Strukturierung beiträgt. Dabei konnten zwei unterschiedliche Verfahren von Scaffolding unterschieden werden, bei denen die Schreiberinnen ihren 
erwachsenen Gesprächspartnern die Übernahme eines Teil ihrer Aufgaben abrangen: FEH ließ sich die richtige Schreibung zahlreicher Grapheme, aber auch einzelner Wörter bestätigen, um den konzeptuellen Prozess nach anfänglichen Unsicherheiten zunehmend nach innen zu verlagern, während SEV sich überwiegend auf den Schreibprozess auf der segmentalen Ebene konzentrierte und die konzeptuelle Arbeit zu großen Teilen dem Interviewer überließ. Dabei erwies sich für FEH die Strategie der Wortsegmentierung mittels Spatien als besonders hilfreich, um zwischen den hierarchiehöheren Ebenen der Textstruktur und den basalen der Phonem-Graphem-Beziehungen hin und her zu wechseln. SEV, die über diese Möglichkeit nicht verfügte, konnte ihren geschriebenen Text dagegen nicht mehr als Orientierung nutzen und die Autorenrolle nur ansatzweise übernehmen.

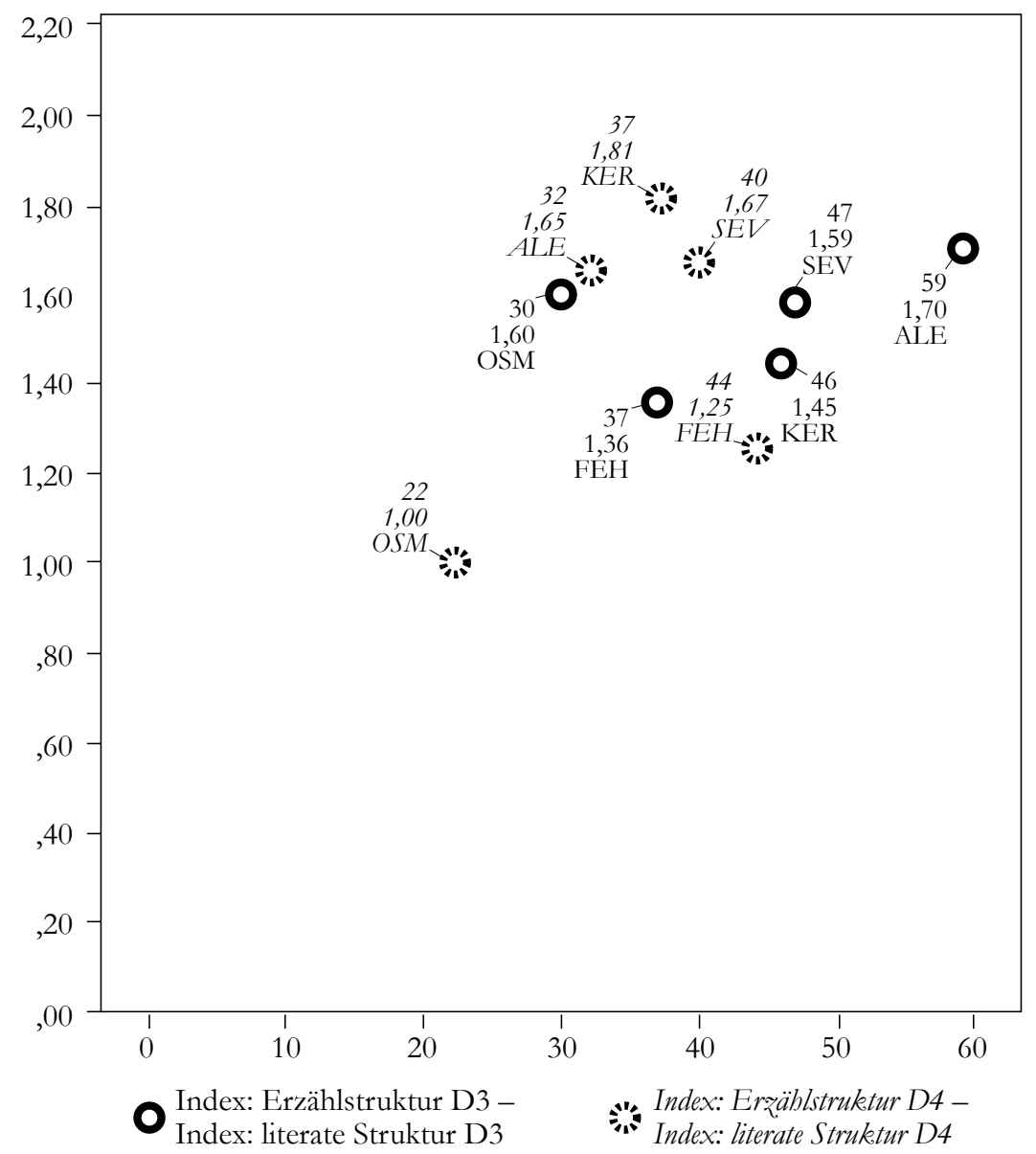

Abb. 2: Gegenüberstellung des literaten und des narrativen Index 
Auch wenn literate und narrative Ausgestaltung tendenziell in dieselbe Richtung wirken, eröffnen sich durch den Gebrauch gesprochener und geschriebener Sprache jeweils unterschiedliche Optionen: Die stärkere narrative Ausgestaltung gelingt vor allem in gesprochener Sprache, während hier beim Schreiben mehr Abstriche in Kauf genommen werden als bei der literaten Strukturierung. In didaktischer Hinsicht stellt die Diktiersituation ein wichtiges Instrument zur Herausforderung der jungen Schreiber durch mediale und konzeptuelle Schriftlichkeit dar, deren Weiterführung ins Schreiben hinein auch im Sinne eines Übergabeprinzips genutzt werden kann, so dass auch für Kinder mit geringeren literalen Ressourcen längere und inhaltlich komplexere Texte möglich werden. Die mediale Komponente des Schriftspracherwerbs könnte mit diesen Prozessen stärker verzahnt werden, indem schon möglichst früh Spatien und Interpunktion für die Gliederung des Textes auf mehreren inhaltlichen und formalen Ebenen genutzt werden, die einen Zugriff auf die jeweilige Medium-FormKorrelation ermöglicht.

\section{Literatur}

Andresen, Helga (2002): Interaktion, Sprache und Spiel: Zur Funktion des Rollenspiels für die Sprachentwicklung im Vorschulalter. Tübingen.

Andresen, Helga (2005): Vom Sprechen zum Schreiben: Sprachentwicklung zwischen dem vierten und siebten Lebensjahr. (= Konzepte der Humanwissenschaften). Stuttgart.

Andresen, Helga (2011): Erlebtes und Fiktives - Zur Dynamik der Entwicklung von Erlebnis- und Phantasiererzählungen im Vorschulalter. In: Hüttis-Graff/Wieler (Hg.), S. 151-180.

Blaschitz, Verena (2010): „Ja grisgot was wollten sie“ - Analyse narrativer Texte 10jähriger Kinder mit Migrationshintergrund. In: Mehlem, Ulrich/Sahel, Said (Hg.): Erwerb schriftsprachlicher Kompetenzen im DaZ-Kontext: Diagnose und Förderung. Freiburg i.Br., S. 93-112.

Becker, Tabea (2001): Kinder lernen erzählen. Zur Entwicklung der narrativen Fähigkeiten von Kindern unter Berücksichtigung der Erzählform. Baltmannsweiler.

Biber, Douglas (1988): Variation across speech and writing. Cambridge u.a.

Boneß, Anja et al. (i.Vorb.): Literacy acquisition in schools in the context of migration and multilingualism. A binational survey.

Boueke, Dietrich et al. (1995): Wie Kinder erzählen: Untersuchungen zur Erzähltheorie und zur Entwicklung narrativer Fähigkeiten. München.

Bühler, Karl (1934): Sprachtheorie. Die Darstellungsfunktion der Sprache. 2., durchges. Aufl. Jena. 
Dehn, Mechthild (2011): Elementare Schriftkultur und Bildungssprache. In: Fürstenau, Sara/Gomolla, Mechtild (Hg.): Migration und schulischer Wandel: Mehrsprachigkeit. Wiesbaden, S. 129-151.

Feilke, Helmuth (2007): Textwelten der Literalität. In: Schmölzer-Eibinger, Sabine/ Weidacher, Georg (Hg.): Textkompetenz. Eine Schlüsselkompetenz und ihre Vermittlung. Festschrift für Paul R. Portmann-Tselikas zum 60. Geburtstag. (= Europäische Studien zur Textlinguistik 4). Tübingen, S. 25-37.

Hargrave, Anne C./Sénéchal, Monique (2000): A book reading intervention with preschool children who have limited vocabularies: the benefits of regular reading and dialogic reading. In: Early Childhood Research Quarterly 15, S. 75-90.

Hausendorf, Heiko/Quasthoff, Uta (2005 [1996]): Sprachentwicklung und Interaktion. Eine linguistische Studie zum Erwerb von Diskursfähigkeiten. Radolfzell. Internet: www.verlag-gespraechsforschung.de/2005/pdf/spracherwerb.pdf. [1. Aufl. 1996 Opladen].

Heath, Shirley B. (1983): Ways with words: language, life, and work in communities and classrooms. Cambridge u.a.

Hüttis-Graff, Petra (2011): Diktierte Texte zu Medienfiguren: Chancen und Grenzen des Diktierens. In: Hüttis-Graff/Wieler (Hg.), S. 207-228

Hüttis-Graff, Petra/Wieler, Petra (Hg.) (2011): Übergänge zwischen Mündlichkeit und Schriftlichkeit im Vor- und Grundschulalter. Freiburg i.Br.

Kern, Friederike/Quasthoff, Uta M. (2005): Fantasy stories and conversational narratives of personal experience: Genre-specific, interactional and developmental perspectives. In: Quasthoff, Uta M./Becker, Tabea (Hg.): Narrative interaction. (= Studies in Narrative 5). Amsterdam u.a., S. 15-56.

Koch, Peter/Oesterreicher, Wulf (1985): Sprache der Nähe - Sprache der Distanz. Mündlichkeit und Schriftlichkeit im Spannungsfeld von Sprachtheorie und Sprachgeschichte. In: Romanistisches Jahrbuch 36, S. 15-43.

Koch, Peter/Oesterreicher, Wulf (2007): Schriftlichkeit und kommunikative Distanz. In: Zeitschrift für Germanistische Linguistik 35, S. 346-375.

Kraus, Karoline (2005): Dialogisches Lesen - neue Wege der Sprachförderung in Kindergarten und Familie. In: Roux, Susanna (Hg.): PISA und die Folgen: Sprache und Sprachförderung im Kindergarten. (= VEP-Aktuell 5). Landau, S. 109-129.

Laufer, Batia/Nation, Paul (1995): Vocabulary size and use: lexical richness in L2 written production. In: Applied Linguistics 16, S. 307-322.

Labov, William (1972): Language in the inner city: studies in the Black English vernacular. (= Conduct and Communication 3). Philadelphia.

Labov, William/Waletzky, Joshua (1967 [1973]): Narrative analysis: oral versions of personal experience. In: Helm, June (Hg.): Essays on the verbal and visual arts. (= Proceedings of the Annual Spring Meeting of the American Ethnological Society 1966). Seattle u.a., S. 12-44. [Dt. Ausg. 1973: Erzählanalyse: mündliche Versionen persönlicher Erfahrung. In: Ihwe, Jens (Hg.): Literaturwissenschaft und Linguistik. Bd. 2. Frankfurt a.M., S. 78-126.] 
Leseman, Paul P.M. et al. (2007): Home literacy as a special language environment to prepare children for school. In: Zeitschrift für Erziehungswissenschaft 10, S. 334 355.

Leseman, Paul P.M. et al. (2009): Bilingual development in early childhood and the languages used at home: competition for scarce resources? In: Gogolin, Ingrid/Neumann, Ursula (Hg.): Streitfall Zweisprachigkeit - The bilingualism controversy. Wiesbaden, S. 289-316.

Lonigan, Christopher J./Whitehurst, Grover J. (1998): Relative efficacy of parent and teacher involvement in a shared-reading intervention for preschool children from low-income backgrounds. In: Early Childhood Research Quarterly 13, S. 263-290.

Ludwig, Otto (2005): Geschichte des Schreibens. Bd. 1: Von der Antike bis zum Buchdruck. Berlin/New York.

Maas, Utz (2008): Sprache und Sprachen in der Migrationsgesellschaft. Die schriftkulturelle Dimension. (= Schriften des Instituts für Migrationsforschung und Interkulturelle Studien der Universität Osnabrück 15). Göttingen.

Maas, Utz (2010): Literat und orat. Grundbegriffe der Analyse geschriebener und gesprochener Sprache. In: Grazer Linguistische Studien 73, S. 21-150.

Maas, Utz/Mehlem, Ulrich (2003): Schriftkulturelle Ressourcen und Barrieren bei marokkanischen Kindern in Deutschland. Unveröff. Manuskript. Osnabrück.

Mehlem, Ulrich (2010): Schreibanlässe und Schreibprozesse in der Grundschule - Literalität durch Interaktion. In: Mehlem, Ulrich/Sahel, Said (Hg.): Erwerb schriftsprachlicher Kompetenzen im DaZ-Kontext: Diagnose und Förderung. Freiburg i.Br., S. $133-160$.

Mehlem, Ulrich (2011): Schriftspracherwerb unter den Bedingungen von Mehrsprachigkeit - Freie Schreibungen von Erstklässlern in Deutsch und Türkisch. In: Hornberg, Sabine/Valtin, Renate (Hg.): Mehrsprachigkeit: Chance oder Hürde beim Schriftspracherwerb? - empirische Befunde und Beispiele guter Praxis. (= DGLSBeiträge 12). Berlin, S. 112-135.

Meng, Katharina/Rehbein, Jochen (Hg.) (2007): Kindliche Kommunikation - einsprachig und mehrsprachig. (= Mehrsprachigkeit 1). Münster u.a.

Merklinger, Daniela (2011): Frühe Zugänge zur Schriftlichkeit. Eine explorative Studie zum Diktieren. Freiburg i.Br.

Ohlhus, Sören (2005): Schreibentwicklung und mündliche Strukturierungsfähigkeiten. In: Feilke, Helmuth/Schmidlin, Regula (Hg.): Literale Textenwicklung: Untersuchungen zum Erwerb von Textkompetenz. (= Forum angewandte Linguistik 45). Frankfurt a.M. u.a., S. 43-68.

O'Loughlin, Kieran (1995): Lexical density in candidate output on direct and semidirect versions of an oral proficiency test. In: Language Testing 12, S. 217-237.

Pohl, Thorsten (2004): Einbettungsstrukturen der Figurenrede im frühen schriftlichen Erzählen. In: Didaktik Deutsch 16, S. 5-14.

Pontecorvo, Clotilde/Zucchermaglio, Cristina (1989): From oral to written language: preschool children dictating stories. In: Journal of Reading Behavior 21, S. 109-126. 
Quasthoff, Uta (1980): Erzählen in Gesprächen. Linguistische Untersuchungen zu Strukturen und Funktionen am Beispiel einer Kommunikationsform des Alltags. (= Kommunikation und Institution 1). Tübingen.

Ravid, Dorit (2006): Semantic development in textual contexts during the school years: Noun Scale analyses. In: Journal of Child Language 33, S. 791-821.

Ravid, Dorit et al. (2002): Subject NP patterning in the development of text production: speech and writing. In: Written Language and Literacy 5, S. 69-94.

Read, John (2000): Assessing vocabulary. (= The Cambridge Language Assessment Series). Cambridge.

Rehbein, Jochen (2007): Erzählen in zwei Sprachen - auf Anforderung. In: Meng/ Rehbein (Hg.), S. 393-459.

Schleppegrell, Mary J. (2004): The language of schooling. A functional linguistics perspective. Mahwah.

Ulich, Michaela (2003): Literacy und sprachliche Bildung im Elementarbereich. In: Weber, Sigrid (Hg.): Die Bildungsbereiche im Kindergarten: Basiswissen für Ausbildung und Praxis. Freiburg i.Br., S. 106-124.

Weinhold, Swantje (2000): Text als Herausforderung. Zur Textkompetenz am Schulanfang. Freiburg i.Br.

Whitehurst, Grover J./Lonigan, Christopher J. (1998): Child development and emergent literacy. In: Child Development 69, S. 848-872.

Wolf, Dagmar/Boueke, Dietrich/Schülein, Frieder (2007): Erzählen nach Bildergeschichten und freies Erzählen. In: Meng/Rehbein (Hg.), S. 155-182.

Wrobel, Arne (2010): Raffael ohne Hände? Mediale Bedingungen und Faktoren des Schreibens und Schreibenlernens. In: Pohl, Thorsten/Steinhoff, Torsten (Hg.): Textformen als Lernformen. (= Kölner Beiträge zur Sprachdidaktik A, 7). Duisburg, S. 27-45. 
